\title{
Reporting harms more transparently in trials of cancer drugs
}

A reference to the CONSORT statement on reporting harms was omitted from the reference list of this article by Bishal Gyawali and colleagues (BMJ 2018;363:k4383, doi:10.1136/ bmj.k4383), resulting in an incorrect citation. The article has been amended to include the reference online. 